\title{
Single azopyridine-substituted porphyrin molecules for configurational and electronic switching $\dagger$
}

\author{
Francesca Matino, ${ }^{* a}$ Guillaume Schull, ${ }^{b}$ Umasish Jana, ${ }^{c}$ Felix Köhler, ${ }^{d}$ Richard Berndt ${ }^{a}$ and \\ Rainer Herges $^{d}$
}

Received 15th April 2010, Accepted 30th July 2010

DOI: $10.1039 / \mathrm{c0cc00959h}$

A new azopyridine functionalized $\mathrm{Ni}$-porphyrin was synthesized as a model compound for deposition and switch on surfaces. Two geometrically and electronically different states of single molecules on $\operatorname{Au}(111)$ were found by scanning tunneling microscopy/ spectroscopy and analyzed with density functional calculations.

Switching is an elementary step in many sophisticated functions, such as directed motion, pumping, information storage and processing, in the macroscopic world as well as at the molecular scale. ${ }^{1}$ For nanoscale applications of such functions, molecules have to be immobilized on solid supports in a well defined geometry and orientation, in order to achieve advanced and reproducible dynamic functions. Moreover, a controlled electronic coupling with the surface as well as sufficient space for molecular movement during an isomerization process are required to avoid charge transfer and hybridization with the surface or intermolecular interactions. ${ }^{2}$ Immobilization and decoupling have been achieved by anchoring functional molecules with a thiol group and an alkane spacer on gold surfaces. ${ }^{3}$ Sterical hindrance within a densely packed selfassembled monolayer can be avoided by dilution of the functional molecules using short alkane thiols. ${ }^{4}$ However, usually stochastic rather than ordered monolayers are obtained. To achieve decoupling from the surface, bulky tert-butyl substituents were attached to a molecular framework to lift it away from the surface. ${ }^{5,6}$ Vertical alignment at a large distance from the surface and control over the distance of the functional groups with respect to each other were attempted by constructing molecular tripods with thiol feet. ${ }^{7}$ Anyway, ordered monolayers are difficult to obtain and it has been shown that not all feet touch the gold surface. ${ }^{7 i}$ Modular platforms and spacers have been recently introduced to control distance from the surface, orientation and packing density. ${ }^{8}$ In view of the construction of controllable functional

${ }^{a}$ Institut für Experimentelle und Angewandte Physik, Christian-Albrechts-Universität zu Kiel, 24098 Kiel, Germany. E-mail:matino@physik.uni-kiel.de; Fax: +49 431880 2510; Tel: + 494318802667

${ }^{b}$ Institut de Physique et Chimie des Matériaux de Strasbourg, UMR 7504 (CNRS - Université de Strasbourg), 67034 Strasbourg, France

${ }^{c}$ Department of Chemistry, Jadavpur University, Kolkata 700032, India.E-mail: jumasish2004@yahoo.co.in

${ }^{d}$ Institut für Organische Chemie, Christian-Albrechts-Universität zu Kiel, 24098 Kiel, Germany

$\dagger$ Electronic supplementary information (ESI) available: Experimental and characterization data for the synthesis of azopyridine functionalized porphyrin. Molecular energy levels from DFT calculation. See DOI: $10.1039 / \mathrm{c} 0 \mathrm{cc} 00959 \mathrm{~h}$ surfaces, further chemical methodologies for a rational vertical design of complex surface architectures are needed.

To combine the cis-trans switching capability of azopyridine with the property of porphyrins to form ordered self-assembled monolayers we covalently attached a 3-azopyridine unit to a $\mathrm{Ni}($ II) tetraphenylporphyrin (Ni-TPP). The porphyrin platform provides bonding to the surface, electronic decoupling and a fixed orientation of the azopyridine unit. Azobenzene functionalized porphyrins have been synthesized previously. However, the azo group was attached in the para position to the meso phenyl substituents, thus lying within the porphyrin molecular plane. ${ }^{9}$ We attached an azopyridine unit in the ortho position to one of the meso phenyl groups of tetraphenylporphyrin, thus allowing the azopyridine to protrude from the surface. Direct synthesis from a mixture of pyrrole, benzaldehyde, and an azopyridine functionalized benzaldehyde in a $4: 3: 1$ ratio failed. Using prefabricated dipyrromethane units for cyclization furnished the monosubstituted tetraphenylporphyrin in $6.6 \%$ yield (Fig. 1, for details see ESI $\dagger$ ). Irradiation of the trans compound with $310 \mathrm{~nm}$ or $520 \mathrm{~nm}$ light in acetonitrile solution gave $15-20 \%$ and $35-40 \%$ cis isomer. Re-isomerisation to the trans compound is achieved by heating to $70-75{ }^{\circ} \mathrm{C}$ for $2 \mathrm{~h}$.

The molecules were deposited from a heated tantalum crucible onto a previously cleaned $\mathrm{Au}(111)$ surface kept at room temperature and investigated by means of a UHV scanning tunnelling microscope (STM) at $5 \mathrm{~K}$. Calculations at the $\mathrm{B} 3 \mathrm{LYP} / 6-311+\mathrm{G}^{*}$ level of density functional theory (DFT) were performed to determine the structure and electronic properties of the molecule, neglecting the substrate.
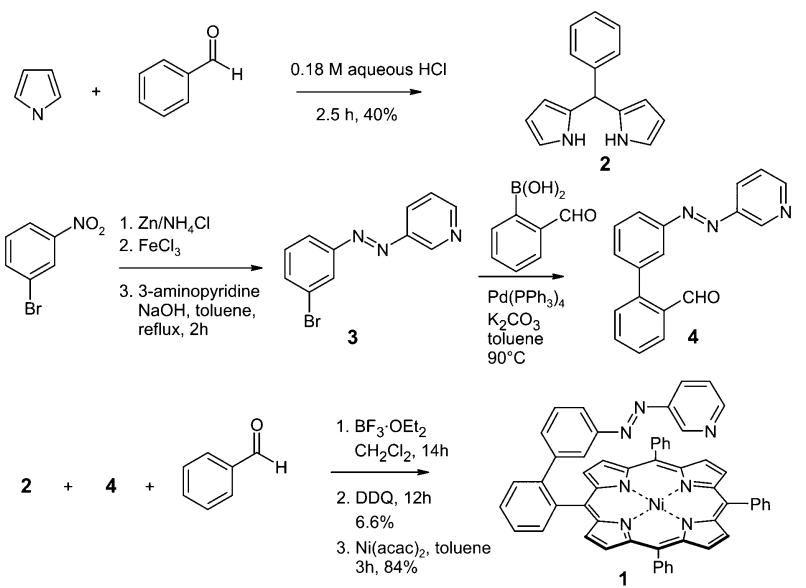

Fig. 1 Synthesis of the azo-functionalized porphyrin 1 (azo/Ni-TPP). 

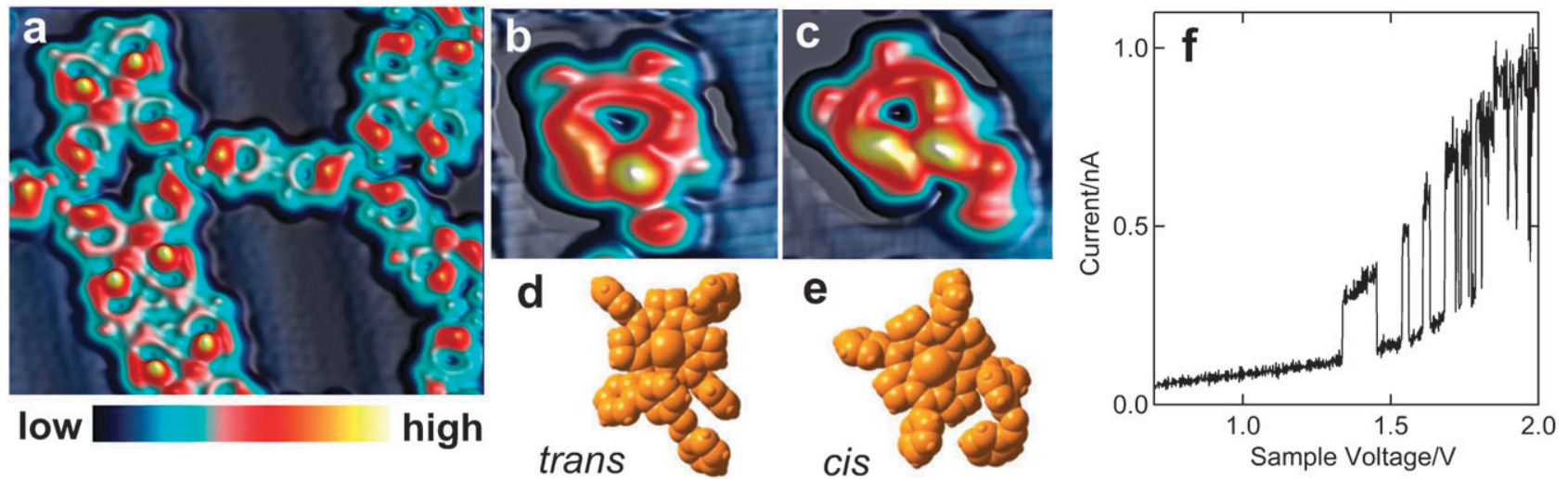

Fig. 2 Pseudo three-dimensional constant current STM images of azo/Ni-TPP adsorbed on Au(111): (a) $12 \times 10 \mathrm{~nm}^{2}$, sample voltage $V=1 \mathrm{~V}$; (b) $2.9 \times 2.9 \mathrm{~nm}^{2}, V=1.4 \mathrm{~V}$; (c) $3.4 \times 2.9 \mathrm{~nm}^{2}, V=1.4 \mathrm{~V}$. (d) and (e) Space filling models of optimized geometries for trans and cis conformations. (f) $I(V)$ characteristic acquired by placing the tip over the azopyridine related protrusion. STM images were treated with a Laplacian filter to enhance small molecular features. ${ }^{10}$

At high coverage, molecules tend to aggregate in long islands aligned along the fcc sites of the herringbone reconstruction. As a result of both porphyrin-porphyrin interaction and hydrogenlike bonds, the islands are composed of trimers and rectangular structures (the lateral dimensions, $1.4 \mathrm{~nm} \times 1.5 \mathrm{~nm}$, are in good agreement with the unit cell parameters reported for Ni-TPP, ${ }^{11}$ Fig. 2(a)). Fig. 2(b) shows a detailed image of a single molecule. Its shape in STM images compares well with the DFT calculated structure of the trans configuration of the azo/Ni-TPP (Fig. 2(d)). The phenyl rings attached to the porphyrin core as well as the azopyridine arm are clearly discernible. ${ }^{12}$ The lower intensity inside the porphyrin ring is assigned to the nickel $\mathrm{d}_{z^{2}}$ filled orbital lying far below the Fermi level. ${ }^{11}$ According to the calculations the phenyl rings and the azo group of the azopyridine unit are pointing away from the surface (i.e. towards the STM tip), which is consistent with the appearance of the latter as a prominent protrusion in STM images (Fig. 2(b)).

As reported in Fig. 2(f), the current acquired over the sidearm of the azo/Ni-TPP can become unstable at sample voltages exceeding $\sim 1.4 \mathrm{~V}$, which may indicate that the arm switches between different conformations. Indeed, controlled manipulation of the arm was achieved by increasing the sample voltage to $\sim 2 \mathrm{~V}$ with the tip positioned over the sidearm of the molecule. Fig. 2(c) shows the molecule from Fig. 2(b) after manipulation. The double lobed azopyridine related feature is now bent and exhibits an additional lobe. Such bending of the azopyridine sidearm is expected for a trans-cis isomerization. The corresponding geometry change is very well represented by the calculations (Fig. 2(d) and (e)). We note that molecules found with a bent sidearm can be switched to a straight arm conformation by applying similar voltages. To further the assignment of trans and cis configurations, scanning tunneling spectroscopy (STS) was performed. The measured differential conductances $\mathrm{d} I / \mathrm{d} V$ of straight (red) and bent (green) sidearm molecules (Fig. 3(a)) may be compared to the calculated densities of states of trans (red) and cis (green) (Fig. 3(b)). The straight and bent arm spectra are markedly different. Clear features at $+1.6 \mathrm{~V},-1.0 \mathrm{~V}$ and $-1.7 \mathrm{~V}$ are visible for the bent configuration. The straight arm configuration exhibits a strong peak at $+1.5 \mathrm{~V}$ and smaller peaks at $-0.9 \mathrm{~V}$ and $-1.2 \mathrm{~V}$. The energies of these peaks are in good agreement with the calculated densities of states for the cis and the trans configurations. The peaks at positive voltage (1.6 V, green curve, and $1.5 \mathrm{~V}$, red curve) can be assigned to the LUMOs of the cis and the trans configurations. The energy difference of these peaks agrees with the calculation, which predicts the LUMO of the trans isomer to be $0.1 \mathrm{eV}$ lower in energy than in cis isomer (Fig. 3(b)). The strong features of the bent configuration at $-1.0 \mathrm{~V}$ and $-1.7 \mathrm{~V}$ are attributed to the first occupied orbitals of the cis isomer, HOMO and HOMO - 1 (mainly located on the porphyrin frame), and the HOMO -2 , which has large coefficients at the lone pairs of the $\mathrm{N}=\mathrm{N}$ group (Fig. 3(b)). For the straight arm configuration, HOMO and HOMO - 1, with small coefficients on the azopyridine arm, are barely discernible in the STS data (low intense features at $-0.9 \mathrm{~V}$ and $-1.2 \mathrm{~V}$, Fig. 3(a)). ${ }^{13} \mathrm{MOs}$ with large coefficients in the trans configured azopyridine arm are very low in energy (HOMO -5 and HOMO -6 ) and beyond the range of the STS measurements (Fig. 3(b), see also Table S1 in ESI $\dagger$ ). The energy difference between HOMO and LUMO in the STS data for the straight arm conformation is reduced by $\sim 0.3 \mathrm{~V}$ with respect to the bent conformation, consistent with the calculation (Fig. 3(a) and (b)). A similar reduction of the energy gap for cis and trans conformers was reported from UV spectroscopy in the parent azobenzene. ${ }^{14}$ The correspondence between the LUMO energies and the voltage instability shown in Fig. 2(f) suggests that these changes occurred in the azo/Ni-TPP as a result of tunneling electrons interacting with the LUMO states. These orbitals are very close in energy in the two configurations, which explains why the switching occurs at similar voltages for both molecules.

A detailed analysis of cis or trans molecules found on the surface after deposition with those resulting from manipulation revealed a slight decrease of their apparent heights in STM images and the switching could not usually be reversed. The observations suggest that the three-dimensional structure of the sidearm is essential for the switching. While an azopyridine arm protruding from the surface may be switched, an arm on the surface leads to significant interaction between the lone pair at the pyridine nitrogen atom with the metal which effectively prevents further switching. ${ }^{15}$ 

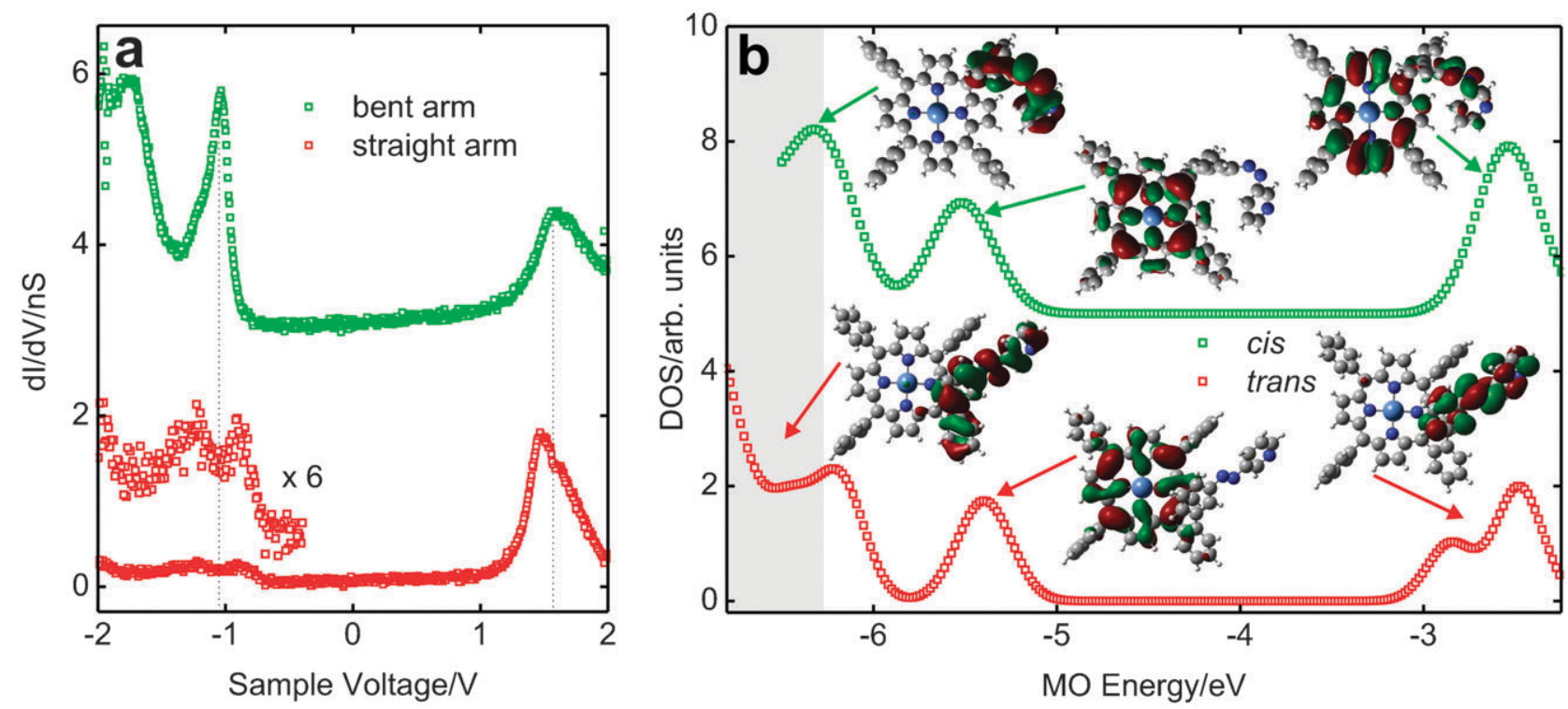

Fig. 3 Electronic structure of the azo/Ni-TPP in the bent azopyridine arm or cis (green) and straight arm or trans (red) configurations. (a) Experimental STS data. STM feedback was opened at $V=2 \mathrm{~V}, I=1 \mathrm{nA}$. The bent arm data are vertically offset for clarity. The dotted lines highlight the reduction of the HOMO-LUMO energy distance between the two conformations. (b) Calculated density of states and most important molecular orbitals. The molecular energy levels are broadened by Gaussian (full width at half maximum $=0.4$ ). The grey area is beyond the voltage range covered by the STS data.

In summary, a new azopyridine functionalized porphyrin was synthesized as a model switch for deposition on surfaces. Two geometrically and electronically different states of single molecules on $\mathrm{Au}(111)$ were found and analyzed by STM and STS. Switching between two conformational states occurs upon tunneling of electrons through LUMO orbitals.

Funding by the Deutsche Forschungsgemeinschaft (SFB 677) and by the Schleswig-Holstein-Fonds is gratefully acknowledged.

\section{Notes and references}

1 Molecular Switches, ed. B. L. Feringa, Wiley, 2001.

2 (a) G. Schull, T. Frederiksen, M. Brandbyge and R. Berndt, Phys. Rev. Lett., 2009, 103, 206803; (b) X. Ge, C. Manzano, R. Berndt, L. T. Anger, F. Köhler and R. Herges, J. Am. Chem. Soc., 2009, 131, 6096; (c) I. F. Torrente, K. J. Franke and J. I. Pascual, J. Phys.: Condens. Matter, 2008, 20, 184001; (d) Y. F. Wang, J. Kröger, R. Berndt and W. A. Hofer, J. Am. Chem. Soc., 2009, 131, 3639.

3 (a) G. Pace, V. Ferri, C. Grave, M. Elbing, C. von Hänisch, M. Zharnikov, M. Mayor, M. A. Rampi and P. Samori, Proc. Natl. Acad. Sci. U. S. A., 2007, 104, 9937; (b) N. Katsonis, T. Kudernac, M. Walko, S. J. van der Molen, B. J. van Wees and B. L. Feringa, Adv. Mater., 2006, 18, 1397; (c) U. Jung, M. Mueller, N. Fujimoto, K. Ikeda, K. Uosaki, U. Cornelissen, F. Tuczek, C. Bornholdt, D. Zargarani, R. Herges and O. Magnussen, J. Colloid Interface Sci., 2010, 341, 366; (d) U. Jung, B. Baisch, D. Kaminski, K. Krug, A. Elsen, T. Weineisen, D. Raffa, J. Stettner, C. Bornholdt, R. Herges and O. Magnussen, J. Electroanal. Chem., 2008, 619-620, 152.

4 (a) Z. J. Donhauser, B. A. Mantooth, K. F. Kelly, L. A. Bumm, J. D. Monnell, J. J. Stapleton, D. W. Price, Jr., A. M. Rawlett, D. L. Allara, J. M. Tour and P. S. Weiss, Science, 2001, 292, 2303; (b) S. Yasuda, T. Nakamura, M. Matsumoto and H. Shigekawa, J. Am. Chem. Soc., 2003, 125, 16430.

5 (a) C. Dri, M. V. Peters, J. Schwaz, S. Hecht and L. Grill, Nat. Nanotechnol., 2008, 3, 649; (b) M. Alemani, M. V. Peters, S. Hecht, K.-H. Rieder, F. Moresco and L. Grill, J. Am. Chem. Soc., 2006, 128, 14446.

6 M. J. Comstock, N. Levy, A. Kirakosian, J. Cho, F. Lauterwasser, J. H. Harvey, D. A. Strubbe, J. M. J. Fréchet, D. Trauner,
S. G. Louie and M. F. Crommie, Phys. Rev. Lett., 2007, 99, 038301 .

7 (a) Q. Li, A. V. Rukavishnikov, P. A. Petukhov, T. O. Zaikova, C. Jin and J. F. W. Keana, J. Org. Chem., 2003, 68, 4862; (b) K. W. Kittredge, M. A. Minton, M.-A. Fox and J. K. Whitesell, Helv. Chim. Acta, 2002, 85, 788; (c) T. Kitagawa, Y. Idomoto, H. Matsubara, D. Hobara, T. Kakiuchi, T. Okazaki and K. Komatsu, J. Org. Chem., 2006, 71, 1362; (d) S. Katano, Y. Kim, H. Matsubara, T. Kitagawa and M. Kawai, J. Am. Chem. Soc., 2007, 129, 2511; (e) H. Jian and J. M. Tour, J. Org. Chem., 2003, 68, 5091; $(f)$ L. Zhu, H. Tang, Y. Harima, K. Yamashita, Y. Aso and T. Otsubo, J. Mater. Chem., 2002, 12, 2250; (g) T. Sakata, S. Maruyama, A. Ueda, H. Otsuka and Y. Miyahara, Langmuir, 2007, 23, 2269; (h) D. Takamatsu, Y. Yamakoshi and K. Fukui, J. Phys. Chem. $B$, 2006, 110, 1968; ( $i)$ L. Wei, H. Tiznado, G. Liu, K. Padmaja, J. S. Lindsey, F. Zaera and D. F. Bocian, J. Phys. Chem. B, 2005, 109, 23963.

8 B. Baisch, D. Raffa, U. Jung, O. M. Magnussen, C. Nicolas, J. Lacour, J. Kubitschke and R. Herges, J. Am. Chem. Soc., 2009, 131, 442.

9 (a) H. Hombrecher and K. Luedke, Tetrahedron, 1993, 49, 9489; (b) C. A. Hunter and L. D. Sarson, Tetrahedron Lett., 1996, 37, 699; (c) S. Tsuchiya, J. Am. Chem. Soc., 1999, 121, 48.

10 I. Horcas, R. Fernandez, J. M. Gomez-Rodriguez, J. Colchero, J. Gomez-Herrero and A. M. Baro, Rev. Sci. Instrum., 2007, 78, 013705.

11 L. Scudiero, D. E. Barlow and K. W. Hipps, J. Phys. Chem. B, 2000, 104, 11899.

12 (a) F. Klappenberger, A. W. Bargioni, W. Auwärter, M. Marschall, A. Schiffrin and J. V. Barth, J. Chem. Phys., 2008, 129, 214702; (b) B.-Y. Choi, S.-J. Kahng, S. Kim, H. Kim, H. W. Kim, Y. J. Song, J. Ihm and Y. Kuk, Phys. Rev. Lett., 2006, 96, 156106.

13 During the acquisition of the STS curves, the tip was placed above the azopyridine arm before the STM feedback was opened. The small coefficients of the HOMO and HOMO - 1 on the sidearm are expected to account for the weak features on the negative side of the $\mathrm{d} I / \mathrm{d} V$ spectrum of the straight configuration.

14 J.-A. Andersson, R. Petterson and L. Tegner, J. Photochem., 1982, 20, 17.

15 At the voltages used in the experiment, no evidence of the trans-cis switching was found for the molecules in the trimeric aggregates. 